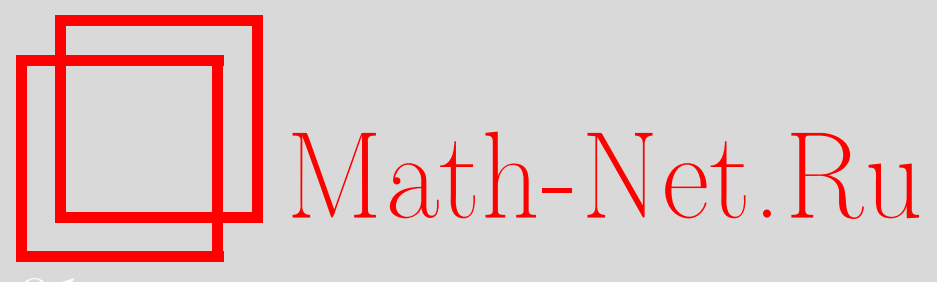

А. Разборов, Чувствительность булевых функций, Квант, 2020, номер 10, 7-16

DOI: https://doi.org/10.4213/kvant20201002

Использование Общероссийского математического портала Math-Net.Ru подразумевает, что вы прочитали и согласны с пользовательским соглашением http: //www . mathnet.ru/rus/agreement

Параметры загрузки:

IP: 54.224 .135 .184

26 апреля 2023 г., $16: 16: 39$

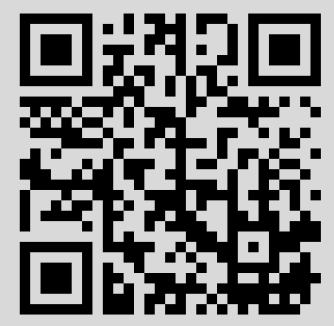




\section{Чувствительность булевых функций}

\section{А. РАЗБОРОВ}

$\mathrm{B}$ ЭТОЙ СТАТЬЕ МЫ РАССКАЖЕМ о красивой и важной задаче с вполне элементарной формулировкой, которая была предметом интенсивных исследований многих математиков (включая автора этой статьи) на протяжении нескольких десятилетий и при этом не поддавалась никаким усилиям. А дальше все произошло как в сказке: 1 июля 2019 года на сайте arxiv.org появилась шестистраничная работа [1] американского математика по имени Хао Хуан (Наo Huang) с полным решением проблемы совершенно неожиданным и удивительно простым способом.

Начнем с азов.

Булевы функции, раскраски гиперкуба и чувствительность. Через $\{0,1\}^{n}$ мы будем обозначать множество всех двоичных слов длины $n$ : например, $\{0,1\}^{3}$ состоит из восьми элементов (000), (001), (010), (011), (100), (101), (110), (111). Булева функиия от $n$ переменных $f_{n}:\{0,1\}^{n} \rightarrow$ $\rightarrow\{0,1\}$ классифицирует слова $x \in\{0,1\}^{n}$ в «хорошие» (те, для которых $\left.f_{n}(x)=1\right)$ и «плохие» $\left(f_{n}(x)=0\right)$. Нижний индекс всегда будет обозначать число переменных.

Булевы функции, как, впрочем, и любые другие, можно подставлять друг в друга; не менее важно то, что их можно подвергать логическим операциям, таким как И, ИЛИ, НЕ. Поэтому булевы функции являются одним из наиболее фундаментальных и часто встречающихся объектов в различных компьютерных науках (и теоретических, и прикладных) как способ

DOI: https://doi.org/10.4213/kvant20201002 представления и преобразования дискретной информации.

Нас сегодня будут больше интересовать свойства булевых функций как комбинаторных объектов, а также иногда их аналитические $^{1}$ свойства; изучением последних занимается целая дисциплина, называемая «анализ булевых функций». Поэтому, чтобы подчеркнуть комбинаторную и геометрическую природу булевых функций, давайте по возможности вместо формул рассматривать картинки и графы.

Два слова $x, y \in\{0,1\}^{n}$ называются соседними, если они отличаются ровно в одной позиции $i \in\{1, \ldots, n\}$. Соединяя ребром все пары соседних вершин, получим граф $Q_{n}$ на $2^{n}$ вершинах. Эти графы (на рисунке 1 показаны графы $Q_{1}, Q_{2}$ и $Q_{3}$ ) называются
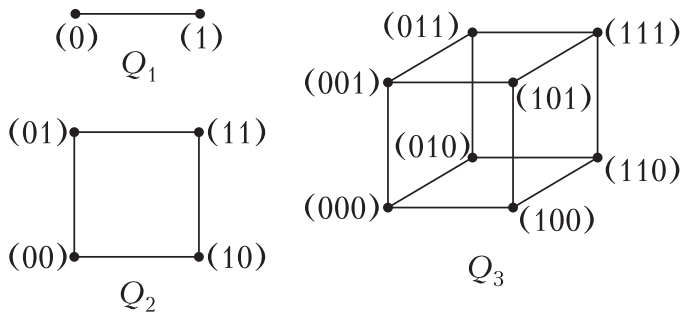

Рис. 1

зиперкубами, и они очень важны в комбинаторике по многим разным причинам. Тем самым булевы функции $f_{n}:\{0,1\}^{n} \rightarrow\{0,1\}$ превращаются в раскраски множества вершин $V\left(Q_{n}\right) n$-мерного гиперкуба в два цвета: зеленый $\left(f_{n}(x)=1\right)$ и красный

1 Здесь стоит напомнить, что 1 и 0 могут рассматриваться не только как логические константы ИСТИНА и ЛОЖЬ, но и просто как вещественные числа. 


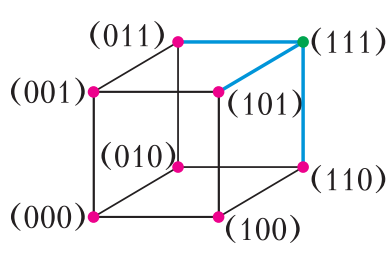

$$
x_{1} \wedge x_{2} \wedge x_{3}
$$
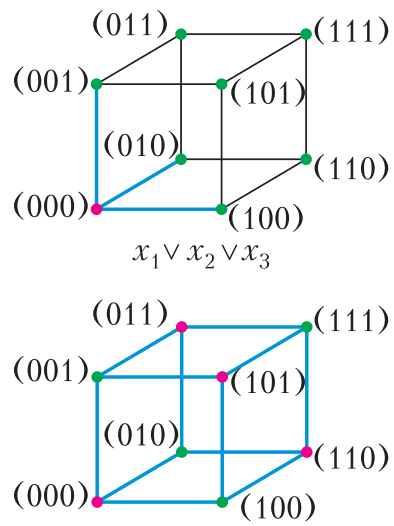

$x_{1} \oplus x_{2} \oplus x_{3}$

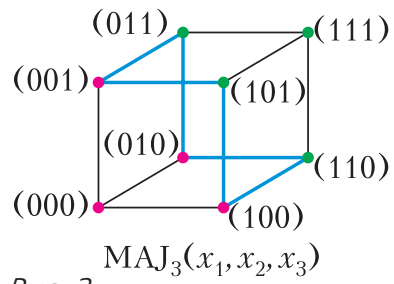

$\begin{array}{ll}\text { Рис. } 2 & \text { функции), и оно } \\ \text { является центральным для нашей статьи }\end{array}$ понятием.

Упражнение 1. Функция $f_{n}$ существенно зави cum om всех своих переменных, если она не может быть выражена как функция от меньшего числа переменных. На нашей картинке это означает, что в ее разрезе имеется хотя бы одно ребро в каждом из $n$ возможных направлений. Функция $f_{n}$ обобщенно симметрична, если ее картинку можно повернуть таким образом, что цвет вершины будет зависеть только от ее уровня, т.е. от количества единиц в соответствующем двоичном слове (рис.3).

Докажите, что всякая функция $f_{2}$, существенно зависящая от обеих своих переменных, является обобщенно-симметричной. А верно ли то же самое для функций от 3 и большего числа переменных?

Мы теперь готовы определить важную числовую характеристику булевой функции $f_{n}-$ ее чувствительность $\mathrm{S}\left(f_{n}\right)$. Начнем с чувствительности $\mathrm{s}\left(f_{n}, x\right)$ функиии $f_{n}$ в данной вериине $x \in V\left(Q_{n}\right):$ по определе-

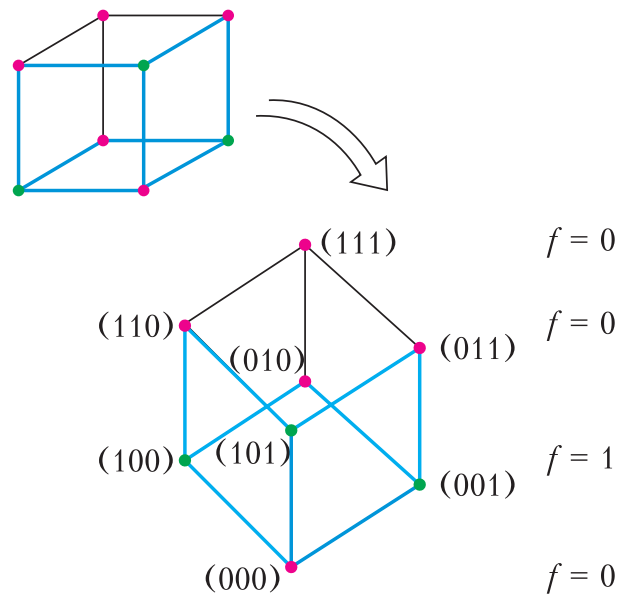

Pис. 3

нию, она просто равна числу ребер в разрезе $\operatorname{Cut}\left(f_{n}\right)$, выходящих из $x$. Это одна из нескольких естественных возможностей измерять, насколько именно функция в точке $x$ чувствительна к малым (в данном случае - ровно в одной координате) изменениям во входных данных. Чувствительность функиии $f_{n}$ определяется как максимально возможное значение $\mathrm{s}\left(f_{n}, x\right)$ :

$$
\mathrm{s}\left(f_{n}\right) \stackrel{\text { def }}{=} \max _{x \in V\left(Q_{n}\right)} \mathrm{s}\left(f_{n}, x\right) .
$$

Таким образом (для знакомых с терминологией теории графов), s $\left(f_{n}\right)$ - это не что иное, как максимальная степень (вершины) графа с множеством ребер $\operatorname{Cut}\left(f_{n}\right)$.

\section{Упражнения}

2. Вычислите $s\left(f_{3}\right)$ для всех функций на рисунке 1.

3. Докажите, что при $n \geq 2$ для любой функции $f_{n}$, существенно зависящей от всех своих переменных, имеет место $\mathrm{s}\left(f_{n}\right) \geq 2$.

Для больших $n$ имеет место оценка

$$
\mathrm{s}\left(f_{n}\right) \geq \frac{1}{2} \log _{2} n-\frac{1}{2} \log _{2} \log _{2} n+\frac{1}{2},
$$

где, как и в упражнении 3 , функция $f_{n}$ существенно зависит от всех своих переменных. Эта оценка, доказанная Х.У.Симоном (H.U.Simon) в 1983 году, по-видимому, является первым нетривиальным результатом в исследовании чувствительности булевых функций. На первый взгляд она может показаться чрезмерно грубой, но на самом деле оценка довольно точна. 
Упражнение 4. Для всех $h \geq 1$ постройте пример функции $f_{h+2^{h}}$, существенно зависящей от $h+2^{h}$ переменных с s $\left(f_{h+2^{h}}\right)=h+1$.

Блочная чувствительность. Понятие чувствительности очень простое и естественное, но оказывается, что большую пользу в различных приложениях приносят его модификации.

Так, например, в формуле (1) вместо взятия максимума по всем $x \in V\left(Q_{n}\right)$ можно взять среднее значение. Получится средняя чувствительность аs $\left(f_{n}\right)$ функции $f_{n}$, которая, как легко понять, является не чем иным, как размером разреза $\left|\operatorname{Cut}\left(f_{n}\right)\right|$, нормированным подходящим (вычислите его!) множителем. Это понятие играет центральную роль в анализе булевых функций.

Нас сегодня, однако, больше интересует другая модификация: bs $\left(f_{n}\right)$, называемая блочной чувствительностью. Как и раньше, начнем со случая, когда у нас есть функция $f_{n}$ и входное слово $x \in\{0,1\}^{n}$. Тогда чувствительным блоком называется подмножество $B \subseteq\{1,2, \ldots, n\}$ такое, что $f\left(x^{B}\right) \neq f(x)$, где $x^{B}-$ результат изменения слова $x$ одновременно во всех позициях $i \in B$. Например, функция $\mathrm{MAJ}_{3}$ на рисунке 1 в точке (000) имеет четыре чувствительных блока, а именно, те подмножества $B \subseteq\{1,2,3\}$, для которых $|B| \geq 2$. Блочной чувствительностью $\operatorname{bs}\left(f_{n}, x\right)$ функиии $f_{n}$ на входе $e^{2}$ называется максимальное значение $l$, для которого существуют $l$ попарно непересекающихся чувствительных блоков. Таким образом, bs $\left(\mathrm{MAJ}_{3}, 000\right)=1$ просто потому, что любые два чувствительных блока имеют непустое пересечение. И, наконец, как и раньше, полагаем

$$
\text { bs }\left(f_{n}\right) \stackrel{\text { def }}{=} \max _{x \in V\left(Q_{n}\right)} \text { bs }\left(f_{n}, x\right) \text {. }
$$

Упражнение 5. Вычислите bs $\left(f_{3}\right)$ для всех функций на рисунке 1.

Ниже мы объясним, почему блочная чувствительность важна и интересна, но

2 Термин «вход» будет иногда использоваться в дальнейшем в качестве сокращения для «входное слово $x \in\{0,1\}^{n} »$. вначале давайте сравним ее с обыкновенной, а также сформулируем гипотезу (уже доказанную), которая послужила поводом для написания этой статьи.

Прежде всего, обыкновенная чувствительность, очевидно, соответствует ограничению, состоящему в том, что все чувствительные блоки имеют размер 1. Поэтому $\mathrm{s}\left(f_{n}, x\right) \leq \mathrm{bs}\left(f_{n}, x\right)$ и, соответственно, $\mathrm{s}\left(f_{n}\right) \leq \mathrm{bs}\left(f_{n}\right)$.

\section{Упражнения}

6. Найдите ошибку в следующем рассуждении. Докажем, что s $\left(f_{n}\right)$ всегда равно bs $\left(f_{n}\right)$. Пусть bs $\left(f_{n}\right)=s$. Рассмотрим соответствующий вход $x$ и систему из $s$ чувствительных блоков на этом входе. Так как ограничение нашей функции на любой блок нетривиально, в каждом блоке существует некоторый вход, на котором функция чувствительна по крайней мере в одной позиции. Так как блоки попарно не пересекаются, эти входы можно между собой соединить и получить вход для исходной функции, чувствительный по крайней мере к $s$ переменным, по одной в каждом блоке. Следовательно, $\mathrm{s}\left(f_{n}\right) \geq s$.

$7^{*}$. Постройте функцию $f_{4}$, у которой $s\left(f_{4}\right)=2$ и $\operatorname{bs}\left(f_{4}\right)=3$.

Насколько большим может быть разрыв между s $\left(f_{n}\right)$ и $\mathrm{bs}\left(f_{n}\right)$ ? Первый общий пример в этом направлении принадлежит Рубинштейну (Rubinstein, 1995). Соответствующую функцию $R_{n}$ от $n=m^{2}$ переменных описать довольно легко, но лучше это делать не на картинке. Итак, у нас есть двоичное слово $x$ длины $m^{2}$. Первым делом разобьем его на $m$ блоков по $m$ знаков в каждом блоке. Назовем блок хорошим, если он содержит ровно две единицы, причем эти единицы следуют друг за другом. Раскрасим $x$ в зеленый цвет, если в нем найдется хотя бы один хороший блок, и в красный, если такого нет.

Упражнение 8. Докажите, что $\mathrm{s}\left(R_{m^{2}}\right)=m$, а bs $\left(R_{m^{2}}\right)=m^{2} / 2$.

Итак, разрыв между чувствительностью и блочной чувствительностью может выражаться квадратичной функцией, и попытки его увеличить оставались совершенно безуспешными в течение долгого времени. У лучшить мультипликативную константу в примере Рубинштейна удалось лишь совсем недавно, но функция при этом так 
и осталась квадратичной. Все это побудило Нисан и Сегеди (Nisan и Szegedy) сформулировать в 1994 году следующую гипотезу, быстро завоевавшую широкую известность.

Гипотеза о блочной чувствительности. Существуют такие абсолютные константы $\varepsilon, \delta>0$, что для любой булевой функции $f_{n}$ имеет место $\mathrm{s}\left(f_{n}\right) \geq \delta \cdot \mathrm{bs}\left(f_{n}\right)^{\varepsilon}$.

Если эту гипотезу сформулировать словами, то в ней утверждается, что функции $\mathrm{s}\left(f_{n}\right)$ и $\mathrm{bs}\left(f_{n}\right)$ совпадают «с точностью до некоторого полинома (многочлена)». Такой уровень точности, в особенности когда полиномиальные функции противопоставляются экспоненциальным, является типичным для современной теории сложности; более подробно об этом можно прочитать в популярном очерке [2].

Достигнутый в течение 25 лет прогресс в доказательстве этого утверждения (или построении к нему контрпримера) преимущественно сводился к нахождению новых гипотез или фактов, напрямую с ним связанных. Приятным исключением является работа Кеньон и Кутина (Kenyon и Kutin) 2004 года, в которой гипотеза о блочной чувствительности доказана в предположении, что все чувствительные блоки имеют ограниченный (например, $\leq 10)$ размер. Мы отсылаем заинтересованного читателя к обзору [3], а сами переходим непосредственно к доказанному Хуаном результату.

Теорема 1 ([1]). Для всякой булевой функчии $f_{n}$ имеем $\mathrm{s}\left(f_{n}\right) \geq \operatorname{bs}\left(f_{n}\right)^{1 / 4}$.

Прежде чем рассказывать об идеях доказательства, давайте выполним данное выше обещание и поговорим о том, почему именно блочная чувствительность важна и интересна. Оказывается, что она «с точностью до полинома» эквивалентна целому ряду других важных характеристик булевых функций. Более того, если читать соответствующие доказательства (до чего мы, к сожалению, не дойдем), то видно, что блочная чувствительность занимает в них центральное место. Мы рассмотрим три такие характеристики, имеющие раз- личную природу: комбинаторную, вычислительную и аналитическую.

Сертификационная сложность. Как всегда, начнем с рассмотрения индивидуального входа $x \in\{0,1\}^{n}$. Множество позиций $I \subseteq\{1, \ldots, n\}$ называется сертифищируюшим (для данного $x$ ), если набор значений $\left(x_{i} \mid i \in I\right)$ (называемый сертификатом) полностью определяет $f_{n}(x)$. Иными словами, для любого другого $y \in\{0,1\}^{n}$, совпадающего с $x$ во всех позициях из $I$, имеет место $f_{n}(y)=f_{n}(x)$. Сертификаты далеко не единственны, и сертификационной сложностью $C\left(f_{n}, x\right)$ называется минимальный (обратите внимание на отличие от определений чувствительности, в которых берется максимум) возможный размер $|I|$ сертификата $I$. После этого, как и раньше,

$$
C\left(f_{n}\right) \stackrel{\text { def }}{=} \max _{x \in\{0,1\}^{n}} C\left(f_{n}, x\right) .
$$

То же самое понятие легко объяснить геометрически. Рассмотрим семейство подкубов в $Q_{n}$, имеющих коразмерность $d$ (т.е. содержащих ровно $2^{n-d}$ вершин). Среди них нас интересуют монохроматические подкубы, т.е. такие, у которых все вершины покрашены в один и тот же цвет.

Упражнение 9. Докажите, что $C\left(f_{n}\right)$ равно минимальному $d$, для которого монохроматические подкубы коразмерности $d$ полностью покрывают $\{0,1\}^{n}$.

Легко понять, что bs $\left(f_{n}, x\right) \leq C\left(f_{n}, x\right)$ и, следовательно, bs $\left(f_{n}\right) \leq C\left(f_{n}\right)$. Действительно, выберем произвольный сертификат $I$ с $|I|=C\left(f_{n}, x\right)$. Тогда всякий чувствительный блок $B$ на входе $x$ обязан содержать хотя бы один $i \in I$. Поэтому всякое дизъюнктное семейство чувствительных блоков (т.е. такое, что никакие два его блока не пересекаются) может содержать не более $|I|$ членов.

В другую сторону это намного менее очевидно.

Теорема 2 (Нисан, Сегеди, 1994). Для всякой булевой функщии $f_{n}$ имеем $C\left(f_{n}\right) \leq \operatorname{bs}\left(f_{n}\right)^{2}$.

Таким образом, bs $\left(f_{n}\right)$ и $C\left(f_{n}\right)$ «эквивалентны с точностью до полинома», и при желании в теореме 1 можно заменить блоч- 
ную чувствительность на сертификационную сложность. От этого ее содержание (за исключением, возможно, константы в экспоненте) не изменится. Отметим также, что в геометрическом определении $C\left(f_{n}\right)$ можно дополнительно потребовать, чтобы искомое покрытие было на самом деле разбиением, т.е. все участвующие в покрытии подкубы попарно не пересекаются. Полученная величина все еще будет полиномиально эквивалентна bs $\left(f_{n}\right)$ и $C\left(f_{n}\right)$; почему это так, станет понятным в следующем разделе.

Разрешающие деревья. До сих пор мы в основном занимались комбинаторными и к тому же локальными (т.е. определяемыми в терминах некоторой «окрестности» фиксированной вершины $\left.x \in\{0,1\}^{n}\right)$ свойствами булевых функций. В этом разделе мы покажем, как все это соотносится с теорией вычислительной сложности.

Разрешающие деревья (decision trees) это, пожалуй, наиболее простая, но в то же время наиболее часто используемая в приложениях вычислительная модель. Многие, вероятно, знают игру в 20 вопросов, в которой требуется угадать неизвестный предмет, задавая про него вопросы, на которые допускаются лишь ответы ДА/НЕТ. Любую мыслимую стратегию для этой игры можно представить в виде разрешаюшего дерева (рис.4). Его высотой называется максимальная возможная длина пути в этом дереве. Таким образом, высота дерева определяется числом вопросов, задаваемых в наиболее неблагоприятном (наихудшем) для нас случае. Напри-

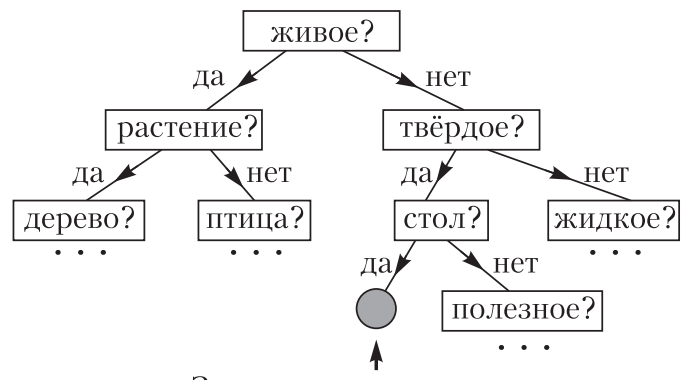

Заключительная вершина

Рис. 4 мер, если на рисунке 4 нам повезло и был загадан именно стол, то все равно высота дерева будет измеряться подсчетом длины вдоль других ветвей этого дерева.

Читателю предлагается обдумать следующий довольно нехитрый тезис: игру в 20 вопросов можно заведомо выиграть тогда и только тогда, когда для нее существует разрешающее дерево, имеющее высоту $\leq 20$.

Как мы уже отмечали, разрешающие деревья в той или иной форме используются везде, и во многих приложениях достаточно рассматривать вычисление с их помощью булевых функций $f_{n}$. Чтобы разобраться в том, что это такое, давайте слегка модифицируем правила игры: предположим, что вместо предмета у нас загадано двоичное слово $x \in\{0,1\}^{n}$. Тогда если ставится задача угадать $x$ полностью, то легко понять, что никакое разрешающее дерево для решения этой задачи не может иметь высоту $\leq n-1$.

Упражнение 10. Докажите это утверждение.

Предположим, однако, что все слово $x$ нас не интересует, а интересует некоторое его свойство, задаваемое булевой функцией $f_{n}(x)$. В такой постановке задача не слишком интересна, потому что про загаданное свойство можно просто спросить. Однако в рассматриваемой нами модели мы разрешаем угадывающему задавать только очень простые вопросы, а именно выяснять значение индивидуального бита $x_{i}(i \in\{1, \ldots, n\})$ в слове $x$. Пример такого разрешающего дерева приведен на рисунке 5; требуется, чтобы информации, полученной угадывающим вдоль каждой ветви, было достаточно для определения зна-

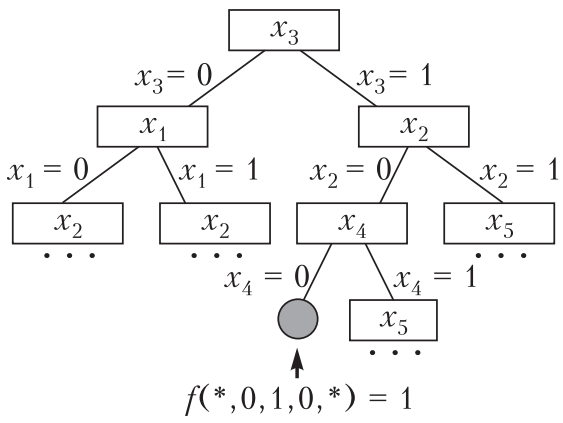

Pис. 5 
чения $f_{n}(x)$ независимо от тех значений, про которые не спрашивали. Сложность $D\left(f_{n}\right)$ булевой функции $f_{n}$ по отношению к разрешающим деревьям (query complexity) определяется как минимально возможная высота разрешающего дерева, вычисляющего нашу функцию. Иными словами, это наименьшее число простых вопросов про вход $x \in\{0,1\}^{n}$, которых заведомо достаточно для вычисления значения $f_{n}(x)$.

\section{Упражнения}

11. Определите $D\left(f_{3}\right)$ для всех функций, изображенных на рисунке 2 .

12. Докажите, что $D\left(f_{n}\right)=D\left(\neg f_{n}\right)$, где $\neg f_{n}-$ отрицание функции $f_{n}$.

13. Докажите, что $D\left(f_{n}\right)=D\left(f_{n}\left(\neg x_{1}, \ldots, \neg x_{n}\right)\right)$.

14. Докажите, что $D\left(f_{n} \vee g_{n}\right) \leq D\left(f_{n}\right)+D\left(g_{n}\right)$. Может ли это неравенство быть строгим?

Теперь давайте свяжем все это с предыдущими рассмотрениями. Как всегда, в одну сторону это просто. Если имеется разрешающее дерево, то каждой заключительной вершине соответствует сертификат, состоящий из ответов на все вопросы, которые угадывающий спросил про $x$ по дороге в эту вершину. На рисунке 5 этот сертификат выписан явно для одной (закрашенной) вершины. Размер этого сертификата не превосходит высоты дерева, и более того, каждый вход $x$ обладает ровно одним таким сертификатом. Таким образом, $C\left(f_{n}\right) \leq D\left(f_{n}\right)$ и, более того (сравните с замечанием в конце предыдущего раздела), построенная нами система подкубов является разбиением $\{0,1\}^{n}$.

В обратную сторону это намного менее очевидно.

Теорема 3 (Билз и др., 2001). Для всякой булевой функиии $f_{n}$ имеем $D\left(f_{n}\right) \leq$ $\leq C\left(f_{n}\right)$ bs $\left(f_{n}\right)\left(\right.$ ито $\leq$ bs $\left(f_{n}\right)^{3}$ ввиду теоремы 2).

Доказательство этой теоремы выходит за рамки этой статьи, но она позволяет добавить разрешающие деревья к списку мер сложности, полиномиально эквивалентных блочной чувствительности. Отметим лишь одно ее замечательное свойство: величины $C\left(f_{n}\right)$, bs $\left(f_{n}\right)$ в правой части имеют сугубо комбинаторную (или геометрическую, если угодно) природу. В то же время разрешающие деревья - это концепция алгоритмическая. Таким образом, теорема 3 утверждает существование алгоритма, качество работы которого целиком определяется комбинаторными свойствами функции, к которой он применяется.

Булевы функции и полиномы. В этом разделе мы рассматриваем 0 и 1 просто как вещественные числа, совершенно абстрагируясь от их логического смысла. Кроме того, нас будет интересовать слегка более общая ситуация $f_{n}:\{0,1\}^{n} \rightarrow \mathbb{R}$, т.е. областью определения функции $f_{n}$ все еще служат вершины гиперкуба, но значениями могут быть уже произвольные вещественные числа.

Мы хотим представить функции $f_{n}$ максимально простым выражением, причем под «представлением» мы имеем в виду, что значение нашего выражения должно совпадать с $f_{n}$ там, где эта функция определена (т.е. при $x \in\{0,1\}^{n}$ ), и может вести себя произвольно на всех остальных.

Самые простые и важные аналитические выражения - это полиномы (многочлены) от нескольких переменных, и оказывается, что в самом деле любая функция $f_{n}:\{0,1\}^{n} \rightarrow \mathbb{R}$ допускает полиномиальное представление. Более того, в таком представлении нет нужды в высших $(\geq 2)$ степенях одной и той же переменной: так как $x_{i} \in\{0,1\}$, то $x_{i}^{d}$ при $d \geq 2$ всегда можно упростить до $x_{i}$. Иными словами, достаточно ограничиться мультилинейными полиномами, т.е. такими, у которых степень по каждой переменной не превосходит 1 (и, следовательно, суммарная степень не превосходит $n$ ).

Упражнение 15. Докажите это утверждение.

Указание. Напишите явную формулу, связывающую $f_{n}\left(x_{1}, \ldots, x_{n}\right) \quad$ с $\quad f_{n}\left(x_{1}, \ldots, x_{n-1}, 0\right)$ и $f_{n}\left(x_{1}, \ldots, x_{n-1}, 1\right)$.

Оказывается, что такое представление к тому же и единственно. Таким образом, всякую функцию $f_{n}:\{0,1\}^{n} \rightarrow \mathbb{R}$ (и, в частности, всякую булеву функцию) можно отождествить с ее мультилинейным полиномиальным представлением. 
Упражнение 16. Представьте в виде мультилинейных полиномов все функции на рисунке 2.

Таким образом, с каждой булевой функцией $f_{n}$ можно связать еще одну меру сложности, на этот раз алгебраическую, а именно степень $\operatorname{deg}\left(f_{n}\right)$ представляющего ее мультилинейного полинома. Отметим ее важное отличие от всех ранее рассмотренных мер. А именно, до сих пор в наши определения входили разнообразные минимумы и максимумы, что на практике ведет к тому, что вычислить их, или даже приблизить разумным образом, может быть непросто. Напротив, степень $\operatorname{deg}\left(f_{n}\right)$ соответствует единственному представлению функции $f_{n}$, и, как следствие, ее можно вычислить явно в большинстве интересных ситуаций, просто выписав представляющий полином.

Видимо, читатель уже догадался, к чему мы ведем: степень $\operatorname{deg}\left(f_{n}\right)$ оказывается эквивалентной всем ранее рассмотренным мерам. Доказать неравенство в одну сторону довольно легко.

Упражнение 17. Докажите, что $\operatorname{deg}\left(f_{n}\right) \leq D\left(f_{n}\right)$.

В другую сторону дело представляется довольно затруднительным: все, что у нас есть - это мультилинейный полином малой степени и заверения в том, что если мы в него подставим 0 или 1, то в результате снова получится 0 или 1. Нам же надо каким-то образом из этого установить, что полученная таким образом булева функция имеет вполне определенную и притом довольно жесткую структуру. На помощь приходит блочная чувствительность: доказывается, что

$$
\operatorname{deg}\left(f_{n}\right) \geq \frac{1}{3} \sqrt{\operatorname{bs}\left(f_{n}\right)} .
$$

Доказательство весьма нетривиально и использует глубокий факт из теории полиномиальной аппроксимации. Грубо говоря, основная идея состоит в том, что bs $\left(f_{n}\right) \geq s-$ это утверждение позитивное, именно этим блочная чувствительность и удобна. Оно означает существование входа $x$ и блоков $B_{1}, \ldots, B_{s}$ с определенными свойствами. Показывается, что наличие таких $x, B_{1}, \ldots, B_{s}$ несовместимо с наличием полиномиального представления малой степени.
В заключение этого раздела отметим, что другой важной характеристикой булевой функции, в особенности при рассмотрении вероятностных и квантовых алгоритмов, является ее приближенная степень $\widetilde{\mathrm{deg}}\left(f_{n}\right)$. Она определяется как минимальная возможная степень мультилинейного полинома $p_{n}\left(x_{1}, \ldots, x_{n}\right)$, для которого $\mid p_{n}\left(x_{1}, \ldots, x_{n}\right)-$ $-f_{n}\left(x_{1}, \ldots, x_{n}\right) \mid \leq 1 / 3$ для всех $x \in\{0,1\}^{n}$ (точное значение константы 1/3 здесь большой роли не играет, важно лишь, что она меньше 1/2). В этом определении уже присутствует минимум, поэтому приближенная степень - намного более сложная величина, чем обыкновенная. Скажем, даже для простой функции на рисунке 6 до-

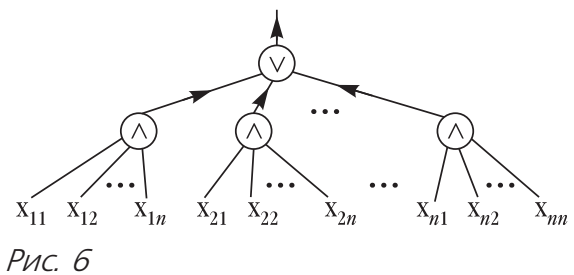

казательство того, что ее приближенная степень линейна по $n$, потребовало многих лет работы и было получено сравнительно недавно специально разработанным для этой цели методом (Бун, Талер (Bun, Thaler), 2013; Шерстов, 2014).

Однако если мы работаем «с точностью до полинома», то приближенная степень пополняет нашу коллекцию мер, полиномально эквивалентных блочной чувствительности. Неравенство $\widetilde{\operatorname{deg}}\left(f_{n}\right) \leq \operatorname{deg}\left(f_{n}\right)$ очевидно, а метод, используемый для доказательства нижней оценки (3) на степень, с тем же успехом работает и для приближенной степени.

Мы познакомились с некоторыми базовыми мерами сложности булевых функций. Несмотря на то, что они имеют совсем разную природу, все они эквивалентны друг другу в некотором разумном смысле, кроме (до 2019 года!) обыкновенной чувствительности. Осталось сказать несколько слов о доказательстве Хуана, лишающем обыкновенную чувствительность ее особого статуса. 


\section{Блочная чувствительность полиноми-} ально эквивалентна чувствительности. Доказательство из [1] существенно опирается на предыдущую работу Готсмана и Линиала (1997), в которой предложена еще одна характеризация блочной чувствительности, и мы начнем с этой работы, тем более что эта часть почти элементарна.

Отправной точкой для нас служит булева функция $f_{n}$ с $\operatorname{deg}\left(f_{n}\right)=d$, а в конце будет показано, что $\mathrm{s}\left(f_{n}\right) \geq d^{1 / 2}$.

Первое наблюдение состоит в том, что без ограничения общности можно считать $d=n$. Для этого достаточно в представляющем $f_{n}$ полиноме выделить произвольный член старшей степени, скажем $\alpha x_{1} x_{2} \ldots x_{d}$ (где $\left.\alpha \neq 0\right)$. Подставим вместо оставшихся переменных $x_{d+1}, \ldots, x_{n}$ произвольные 0-1 значения (например, нули). Легко понять, что от такой операции степень полинома уменьшиться не может, просто потому что член $\alpha x_{1} \ldots x_{d}$ мы не трогаем и сокращаться ему будет не с кем. С другой стороны, чувствительность (как, впрочем, и все остальные рассмотренные в этой статье меры) от такой подстановки может только уменьшиться.

Упражнение 18. Докажите это утверждение.

Итак, предположим, что старший член $x_{1} x_{2} \ldots x_{n}$ присутствует в $f_{n}$ с ненулевым коэффициентом $\alpha$. Оказывается, что $\alpha$ имеет очень полезное комбинаторное описание. Развернем наш гиперкуб, чтобы на каждом горизонтальном уровне оказались входы с одинаковым количеством единиц, и поменяем цвет вершин с красного на зеленый и наоборот, но только на нечетных уровнях (рис.7).

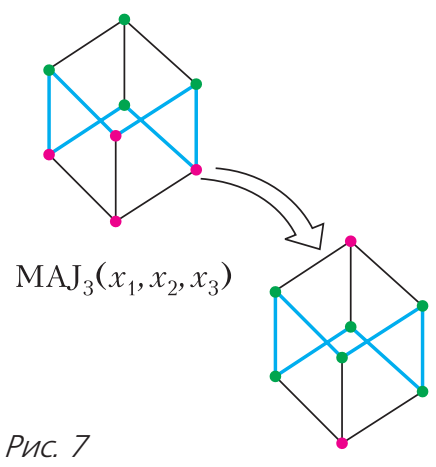

Что произойдет от такой операции? Вопервых, разрез $\mathrm{Cut}\left(f_{n}\right)$ превратится в множество монохроматических ребер, т.е. таких, у которых оба конца покрашены в один цвет. Тем самым, если мы рассмотрим подграфы $G_{n}, R_{n}$, индуцированные зелеными и красными (в новой раскраске!) вершинами соответственно, то $\mathrm{S}\left(f_{n}\right)$ станет не чем иным, как $\max \left(\Delta\left(G_{n}\right), \Delta\left(R_{n}\right)\right)$, где $\Delta(G)$ максимальная степень вершины в графе $G$.

Оказывается, что коэффициент $\alpha$ просто измеряет «несбалансированность» нашей новой раскраски. Более точно, если $g_{n}=\left|V\left(G_{n}\right)\right|$ и $r_{n}=\left|V\left(R_{n}\right)\right|-$ количества «новых» зеленых и красных вершин, то имеет место соотношение

$$
g_{n}-r_{n}=\alpha \cdot 2^{n} .
$$

Для нас единственно важно, что $g_{n} \neq r_{n}$, т.е. что одно из этих чисел (скажем, $g_{n}$ ) строго больше $2^{n-1}$.

Таким образом, нам «всего лишь» осталось показать, что для произвольного подмножества $V_{n} \subseteq\{0,1\}^{n}$ c $\left|V_{n}\right|>2^{n-1}$ в индуиированном этим подмножеством графе $H_{n}$ есть вериина степени $\geq n^{1 / 2}$.

Упражнение 19. Покажите, что это утверждение станет неверным, если неравенство $\left|V_{n}\right|>2^{n-1}$ заменить на нестрогое.

Это и есть сведение Готсмана-Линиала. Для полноты картины отметим, что оно работает в обе стороны, и если в выделенном курсивом утверждении заменить $n^{1 / 2}$ на $n^{\varepsilon}$ для произвольной константы $\varepsilon$, то получится еще одна переформулировка гипотезы о блочной чувствительности.

Итак, нижняя оценка на максимальную степень $H_{n}$ и есть то, что доказывает Хуан. Доказательство несложно для понимания, но использует некоторые элементарные (на уровне первого курса любого математического факультета) факты из линейной алгебры. Строго их формулировать, а тем более доказывать, у нас возможности нет, но некоторое представление, о чем идет речь, мы дать можем.

Матрищей называется квадратная таблица $A$ размера $N \times N$, состоящая из чисел $a_{i, j}(1 \leq i \leq N, 1 \leq j \leq N)$. Матрица $A$ называется симметричной, если $a_{i, j}=a_{j, i}$ для 


$$
\begin{gathered}
A=\left(\begin{array}{ccc}
1 & 2 & -2 \\
2 & 1 & -2 \\
-2 & -2 & 1
\end{array}\right) \quad X=\left(\begin{array}{c}
1 \\
1 \\
-1
\end{array}\right) \\
A X=\left(\begin{array}{c}
5 \\
5 \\
-5
\end{array}\right) \\
X^{1}=\left(\begin{array}{c}
1 \\
1 \\
-1
\end{array}\right) \quad \lambda_{1}=5 \\
X^{2}=\left(\begin{array}{l}
1 \\
0 \\
1
\end{array}\right), \quad X^{3}=\left(\begin{array}{c}
1 \\
-1 \\
0
\end{array}\right) \\
\lambda_{2}=\lambda_{3}=-1
\end{gathered}
$$

Pис. 8

всех $i, j$. На картинке это означает, что матрица не изменится при отражении относительно главной диагонали, идущей из левого верхнего в правый нижний угол (рис.8). В оставшейся части статьи нас будут интересовать исключительно симметрические матрицы.

Вектор размерности $N$ - это столбец $X$ высоты $N$, также состоящий из чисел $x_{j}$ $(1 \leq j \leq N)$. Матрицу $A$ размера $N \times N$ всегда можно умножить на вектор той же размерности $N$; получится новый вектор, который обозначается через $A X . \mathrm{B} i$-й позиции этого вектора помещается значение суммы $a_{i, 1} x_{1}+\ldots+a_{i, N} x_{N}$; пример такого вычисления приведен на рисунке 8 .

Этот пример интересен еще и потому, что вектор $A X$ оказывается гомотетичен исходному вектору $X$, т.е. существует такое число $\lambda$, что $(A X)_{i}=\lambda x_{i}$ для всех $1 \leq i \leq N$, или, в векторных обозначениях, $A X=\lambda X$. Векторы, обладающие этим замечательным свойством, называются собственными (для матрицы $A$ ), а «коэффициент гомотетии» ${ }^{3} \lambda-$ собственным значением, соответствующим собственному вектору $X$. У каждой матрицы есть тривиальный собственный вектор, а именно, состоящий из одних нулей нулевой вектор, но на первый взгляд непонятно, почему у $A$ непременно должны быть какие-то другие.

На самом деле у произвольной (т.е. не обязательно симметрической) матрицы их

\footnotetext{
${ }^{3}$ Коэффициент $\lambda$ вполне может быть нуле-
} вым или отрицательным! вполне может и не оказаться, и для полного понимания картины необходимо привлекать комплексные числа. Но для симметрических матриц все существенно проще: всякая такая матрица обладает полным набором $X^{1}, \ldots, X^{N}$ линейно независимых (см. следующий абзац) собственных векторов. Так, например, наша матрица $A$ на рисунке 8 , помимо уже известного нам собственного вектора $X=X^{1}$ обладает еще двумя, $X^{2}$ и $X^{3}$. Тем самым у нас также появляется набор собственных значений $\lambda_{1}, \ldots, \lambda_{N}$, соответствующих этим собственным векторам, причем (все это надо доказывать, конечно) с точностью до перестановки этот набор зависит только от матрицы $A$ и называется ее спектром. В нашем примере спектр матрицы состоит из чисел $(5,-1,-1)$; обратите внимание, что в нем появляются не только отрицательные числа, но и кратные значения, т.е. одному и тому же собственному значению могут соответствовать несколько (линейно независимых!) собственных векторов.

В заключение нашего краткого экскурса в линейную алгебру скажем, как мы уже обещали, несколько слов про теорию размерности. Векторы $X^{1}, \ldots, X^{M}$ размерности $N$ называются линейно независимьми, если не существует никакой нетривиальной (т.е. такой, в которой хотя бы одно из $\alpha_{i}$ отлично от 0 ) линейной комбинации $\alpha_{1} X^{1}+\ldots+\alpha_{M} X^{M}$, равной нулевому вектору. Число линейно независимых векторов $M$ в такой системе не превосходит размерности $N$, и в случае $M=N$ система линейно независимых векторов называется базисом. Базисы можно также охарактеризовать в противоположных (или, как часто говорят математики, двойственных) терминах. Именно, система $X^{1}, \ldots, X^{N} N$-мерных векторов является базисом тогда и только тогда, когда любой другой $N$-мерный вектор $Y$ можно выразить в виде линейной комбинации $\alpha_{1} X^{1}+\ldots+\alpha_{N} X^{N}$.

\section{Упражнения}

20. Докажите, что если $X^{1}, \ldots, X^{N}$ - базис, то всякий $N$-мерный вектор обладает единственным представлением вида $\alpha_{1} X^{1}+\ldots+\alpha_{N} X^{N}$. 
21. Проверьте, что векторы $X^{1}, X^{2}, X^{3}$ на рисунке 8 образуют базис.

Вернемся к доказательству выделенного курсивом утверждения перед упражнением 19. В качестве первого шага Хуан конструирует специальную симметрическую матрицу $A_{n}$ размера $N \times N$, где $N=2^{n}$, строки и столбцы которой занумерованы всеми вершинами гиперкуба $x, y$ (а не только элементами $V_{n}$ ). Все элементы в этой матрице равны 0,1 или -1 , причем $A_{n}(x, y)=0$ тогда и только тогда, когда $x$ и $y$ не соединены ребром (в частности, $A_{n}(x, x)=0$ на диагонали).

Если бы вхождений -1 в матрице $A_{n}$ не было вообще, то она называлась бы матрицей смежности (гиперкуба); это очень важный объект в теории графов. Ее спектр хорошо известен, но он не тот, что нам нужен. Путем замены 1 на -1 в правильным образом выбранных местах удается добиться того, что спектр будет состоять ровно из $N / 2$ значений $n^{1 / 2}$ и $N / 2$ значений $-n^{1 / 2}$.

Далее, множество вершин $V_{n}$ определяет симметрическую подматрицу $B_{n}$ в $A_{n}$. Эта подматрица имеет большой размер, и мы хотим вывести отсюда какое-нибудь нетривиальное заключение про ее спектр. Слегка упрощая доказательство Хуана, рассмотрим систему, состоящую из $\mathrm{N} / 2$ линейно независимых собственных векторов $X^{1}, \ldots, X^{N / 2}$ матрицы $A_{n}$, соответствующих собственному значению $n^{1 / 2}$, а также векторов $Y^{v}$, для каждого $v \in V_{n}$, в которых в $v$-й позиции стоит 1 , а все остальные позиции заполнены нулями. Векторов в этой системе строго больше, чем $N$, поэтому базисом она быть не может. Следовательно, существует нетривиальное равенство вида $\alpha_{1} X^{1}+\ldots+\alpha_{N / 2} X^{N / 2}+\sum_{v \in V_{n}} \beta_{v} Y^{v}=0$; легко понять (проверьте!), что тогда $\sum_{\text {vх } V_{n}} \beta_{v} Y^{v}$ будет нетривиальным собственным вектором матрицы $B_{n}$, и ему все еще соответствует собственное значение $n^{1 / 2}$.

Наконец доказывается, что максимальная степень в графе $H_{n}$ не меньше, чем любое собственное значение матрицы $B_{n}$. Если бы отрицательных вхождений -1 в матрице $B_{n}$ не было (т.е. $B_{n}$ была бы просто матрицей смежности), то этот факт широко известен и не менее широко применятся в теории графов. Замена некоторых 1 на -1 этому рассуждению только помогает, и это легко проверяется.

Упражнение 22. Пусть $G$ - произвольный граф с множеством вершин $\{1,2, \ldots, N\}$ и $A$ - произвольная симметрическая матрица размера $N \times N$ такая, что $a_{i, j}=0$, если $i$ и $j$ не соединены ребром, и $a_{i, j}= \pm 1$, если соединены. Докажите, что всякое собственное значение $\lambda$ матрицы $A$ не превосходит максимальной степени $\Delta(G)$ графа $G$.

Вот собственно и все.

В заключение хочется отметить, что важные математические открытия происходят по-разному. Бывает, что решение требует построения глубоких и важных теорий, усилий нескольких поколений математиков и т.д. А бывает, как в нашем случае, что все, что для этого требуется, - это одна красивая и неожиданная идея. Прелесть математики (одна из многих) состоит в ее непредсказуемости: предугадать развитие событий заранее совершенно невозможно.

\section{Литература}

1. H.Huang. Induced subgraphs of hypercubes and a proof of the sensitivity conjecture. - Annals of Mathematics, 190(3):949-955, 2019.

2. А.А.Разборов. Теория сложности. В книге: Математическая составляющая, 2-е изд. - М.: Математические этюды, 2019.

3. P.Hatami, R.Kulkarni, and D.Pankratov. Variations on the sensitivity conjecture. - Theory of Computing Library, Graduate Surveys, 4:1-27, 2011. 